\title{
Eight drive-reward combinations: A test of incentive-motivational theory
}

\author{
W. MILES COX \\ University of South Carolina, Columbia, South Carolina 29208
}

\begin{abstract}
Eight groups of 12 rats each were trained to traverse a runway under one of four drive-reward combinations (deprived-food, deprived-no food, satiated-food, or satiated-no food) and then were shifted to a different combination. During Phase I, only deprived-food groups showed increasing response speeds, and no energizing effect of drive was obtained for the deprived-no food group. During Phase II, the group which continued to show some consummatory activity even though it was food satiated extinguished more slowly than the groups from which food reward was omitted. For the other groups, "satiated" or nonrewarded training during Phase I had no effect upon performance during Phase II. The results of both phases of the experiment support the view that consummatory activity is the necessary event in instrumental reward conditioning.
\end{abstract}

Spence (1956) assumed that a major component of instrumental appetitive conditioning is the development of incentive motivation $(K)$ which combines with drive $(\mathrm{D})$ and habit $(\mathrm{H})$ to determine performance $(\mathrm{E})$ : $\mathrm{E}=\mathrm{H} \times(\mathrm{D}+\mathrm{K})$. Black (1965) has further asserted that $\mathrm{K}$, a consequence of the classically conditioned $\mathrm{r}_{\mathrm{g}}-\mathrm{sg}$ mechanism, will accrue only in situations which allow overt consummatory activity $\left(R_{g}\right)$ to be elicited, so that the joint occurrence of deprivation and a consummable reward is necessary for $K$ to develop. The latter analysis also implies that extinction of $r_{g}-s_{g}$ through the nonelicitation of $R_{g}$ is responsible for decreasing response speeds following removal of reward.

Black's (1965) analysis was prompted by a series of experiments by Seward and his associates (Seward, Shea, \& Elkind, 1958; Seward \& Procter, 1960; Seward, Shea, \& Davenport, 1960) which purported to find, contrary to Spence's (1956) prediction of an additive rather than a multiplicative relationship between $\mathrm{D}$ and $\mathrm{K}$, an interaction of these two theoretical variables. Black (1965) suggested that the apparent interaction reported by Seward et al. resulted from an incorrect designation of nondeprived animals which received a food reward as "high K" subjects. Since these nondeprived subjects presumably ate little or none of their available food in the goalbox, $\mathrm{K}$ did not accrue for them and performance would not be expected to improve any more than for deprived subjects for which no reward was available. Since, however, Seward et al. $(1958,1960,1960)$ failed to present extended conditioning curves,

This paper is sponsored by Roger W. Black. The research was conducted while the author was an NDEA Predoctoral Fellow at the University of South Carolina. Requests for reprints should be addressed to the author who is now at the Department of Psychology, Indiana University, Bloomington, Indiana 47401.
Black's conjecture with regard to the lack of improvement for "satiated" subjects remained largely that.

The present experiment involved the same four drive-reward combinations-deprived-food (DF), deprived-no food (DNF), satiated-food (SF), and satiated-no food (SNF) - which had been employed in the Seward et al. studies, but extended training showing the full course of the learning process was given in order to test Black's (1965) assumptions. The more novel aspect of the experiment involved shifting groups trained under one of the four drive-reward combinations to a different one of these combinations for a second phase of training. The basic design involved the factorial arrangement of the four drive-reward combinations with themselves to yield 16 preshift-postshift arrangements. The nonshifted combinations DF-DF, DNF-DNF, SF-SF, and SNF-SNF) were excluded, however, because each of the drive-reward conditions was represented in Phase I by combinations which were included for other purposes; four other combinations (DNF-SNF, SF-SNF, SNF-DNF, and SNF-SF) were also eliminated because they would have involved satiation and/or nonreward during both phases, and thus groups receiving such training would be expected to show no substantial changes in performance during either phase. The remaining combinations allowed response acquisition to be studied under the four drive-reward conditions during Phase I (e.g., DF-DNF, DNF,-DF, SF-DF, and SNF-DF); response elimination was studied under several different conditions during Phase II after acquisition under the DF combination during Phase I (i.e., DF-DNF, DF-SF, and DF-SNF); finally, the DNF-DF, SF-DF, SNF-DF, and the SF-DNF, DNF-SF combinations indicated the effects of initial satiation and/or nonreinforcing training procedures upon performance 
under other drive-reward combinations during Phase II. These eight combinations which were of major interest comprised, then, an "incomplete" factorial.

\section{METHOD}

\section{Subjects}

The study was conducted in two independent replications with a total of 96 female albino rats of the Sprague-Dawley strain. Replication I consisted of 64 animals, 85 days old at the beginning of training. which were obtained from the Sprague-Dawley Company, Madison, Wisconsin, and in Replication II there were 32 animals, 80 days old at the beginning of training, which were obtained from Cherokee Farms of Atlanta, Georgia.

\section{Apparatus}

The apparatus was a standard straight runway whose interior was $116.84 \mathrm{~cm}$ in length and which was partitioned into a $25.40-\mathrm{cm}$ startbox, a $60.96-\mathrm{cm}$ alley, and a $30.48-\mathrm{cm}$ goalbox; the interior of all sections was $8.99 \mathrm{~cm}$ wide and $9.32 \mathrm{~cm}$ high. The alley and goal sections were painted flat black and the startbox was painted flat gray; the top of each division consisted of a hinged section of clear Plexiglas. Running time was measured from the startbox door to a point $50.80 \mathrm{~cm}$ within the alley, and goal time was measured from that point to a point which was $20.32 \mathrm{~cm}$ within the goalbox, a total distance of $30.48 \mathrm{~cm}$.

\section{Pretraining procedure}

Subjects were housed in individual home cages where they had an ad-lib supply of food and water until 11 days prior to training when all food was removed. On the succeeding 10 days, a deprivation diet consisting of $9 \mathrm{~g}$ of Purina Lab Chow was provided daily, but an ad-lib supply of water continued to be available in both the home cages and carrying cages throughout the experiment. On each day of the pretraining deprivation period, subjects were handled in groups of four animals for $10 \mathrm{~min}$ or individually for approximately $2 \mathrm{~min}$, and on the day immediately preceding training, the subjects explored the apparatus in groups of four animals for $10 \mathrm{~min}$ each.

\section{Deprivation and Satiation Procedures}

During experimental training, all groups were provided with the same amount of maintenance food each day, with the satiated or deprived conditions being maintained by providing subjects with their daily food either before or after their daily trials were administered. This procedure was instituted to maintain equivalent body weights among the groups in order that performance (viz., that of Groups DNF-DF, SF-DF, and SNF-DF) during Phase II would not be confounded with weight differences which might have developed during Phase I. To test the adequacy of this procedure, the body weights were sampled at various points during the training period.

In an attempt to produce as complete food satiation as possible, within the above limitation, subjects in the satiated groups were given in their home cages $1 \mathrm{~h}$ before a squad received its daily trials a large supply of both Noyes food pellets and a "palatable" wet mash which consisted of dry ingredients-four parts of granulated Lab Chow, one part of sucrose, and five parts of powdered milk-mixed with water. The pellets and mash were also available in the carrying cages during training, but as soon as the daily trials were completed, all food was removed until $1 \mathrm{~h}$ before training was initiated on the following day. Subjects in the deprived groups were given the pellets and wet mash in their home cages immediately after their daily trials for a period equal to that for which the satiated groups had such access (the $1 \mathrm{~h}$ for satiation plus the time required to run the daily trials, or a total of approximately $2 \mathrm{~h}$ $15 \mathrm{~min}$ ). These subjects were thus under approximately $201 / 2 \mathrm{~h}$ of deprivation at the time their trials were initiated on the following day.

\section{Experimental Training}

The subjects were run in squads of 16 animals, and each squad was run at the same time each day with the first squad being given all of its daily trials before the second squad was run, etc. Within a squad, each subject received its first daily trial before any subject received its second trial, etc. This procedure resulted in an intertrial interval of 15-20 min.

During Phase I, two trials were administered on the first 2 days of training, and thereafter there were four daily trials, for a total of 60 trials for Phase I. A trial consisted of placing the subject into the startbox facing away from the startbox door. As soon as the subject had oriented toward the startbox door, it was opened, and as soon as the subject had completely entered the alley, the door was reclosed to prevent reentry. When the subject had entered the goalbox, the goalbox door was also closed to prevent retracing. For each of the "rewarded" (DF and SF) groups, five 45-mg Noyes food pellets were placed into a food dish in the goalbox, while the unbaited food dish was placed into the goalbox for the nonrewarded (DNF and SNF) groups. The subject was removed from the goalbox and returned to its carrying cage after a 20 -sec detention in the goalbox. If a subject failed to traverse the alley before $120 \mathrm{sec}$ had elapsed, it was prodded to the goalbox, and $120 \mathrm{sec}$ was arbitrarily recorded for running time and for goal time. Phase II was begun on the day immediately following completion of Phase $I$, with each group receiving the appropriate shift in drive and/or reward. During this phase, four trials were administered each day for 15 days, for a total of 60 trials.

\section{RESULTS AND DISCUSSION}

Prior to analysis, latencies were converted into speeds (one/time) which in turn were combined into blocks of four trials each. Since graphical representations indicated no systematic differences in the two replications, the data were collapsed for presentation and analysis, and since running and goal speeds yield similar information, only the latter are presented here. Preliminary analyses also indicated that body weights of the groups for which it was necessary to maintain equivalent levels of drive during Phase II were not reliably different on any of the days on which the weights were sampled.

\section{Phase I}

Inspection of Figure 1 indicates that differences developed in the performance of the eight groups during Phase I. Thus, the three groups which were both deprived and rewarded (DF-DNF, DF-SNF, and DF-SF) showed consistent increases in response speeds, and these groups reached the same asymptotic level of performance. On the other hand, the performance of the groups which were either satiated or nonrewarded, or both, declined gradually throughout Phase I, while these five groups did not differ from each other. These observations are confirmed by statistically reliable effects for trial blocks for both comparisons, $F(14,420)=85.66$, $\mathrm{p}<.001$, and $\mathrm{F}(14,700)=7.09, \mathrm{p}<.001$, respectively, and by nonreliable effects for groups, $F<1.0$ in both cases.

Since only subjects in the DF groups ate in the goalbox, and since only these groups exhibited 
increasing response speeds, the results for Phase $I$, in short, are entirely consistent with the view that there should be no increase in response speeds unless overt consummatory responses are made in the goalbox, regardless of the presence or absence of food (Black, 1965). It should be noted further that subjects which were trained under the high level of drive and without reward (Group DNF) did not reflect a "general energizing" effect of D (Spence, 1956) since they did not perform at a higher level than the satiated subjects (SF and SNF). Since no such effect for $D$ was obtained, the results for Phase $I$ in addition to indicating the influence of $D$ upon the development of $K$ also support the suggestion that $D$ facilitates performance only to the extent that it enhances consummatory activity (Black, 1969, p. 132).

\section{Phase II}

Examination of the performance of the eight groups during Phase II (Figure 2) indicates three different patterns of responding. Thus, the groups which first encountered the combination of both deprivation and reward in Phase II (DNF-DF, SF-DF, and SNF-DF) showed rapidly improving performance during that phase. On the other hand, the groups which were deprived and rewarded in Phase I but were shifted to satiation or nonreward, or both, in Phase II showed rapidly declining performance during that phase. These effects in both cases were confirmed statistically by reliable effects for trial blocks, $F(14,420)=77.57$, $\mathrm{p}<.001$, and $\mathrm{F}(14,420)=42.94, \mathrm{p}<.001$, respectively. Finally, those groups which were trained under satiation or nonreward in Phase $I$, and which were continued under one of these conditions in Phase II, showed a level of performance during Phase II which was similar to the poor performance they had shown at the end of Phase I.

Response elimination. Analysis of variance of the goal speeds during Phase II for the three groups which were trained under both deprivation and reward in Phase I and which were shifted to satiation or nonreward or both in Phase II indicated that these groups differed reliably, $F(2,33)=5.25, p<.05$. Individual comparisons (Duncan's range test) showed that Group DF-SF differed from Group DF-SNF $(p<.05)$; that the difference between Groups DF-SF and DF-DNF was nearly significant $(.05<\mathrm{p}<.055)$; but that Groups DF-DNF and DF-SNF did not differ $(p>.10)$. The consummatory activity data of Group DF-SF indicate that subjects in this group continued to eat during Phase II, but that there was a progressive decrement in the number of pellets consumed during the course of testing under satiation.

The fact that Groups DF-DNF and DF-SNF, which received the usual extinction procedure in which reward is removed from the goalbox, showed the most

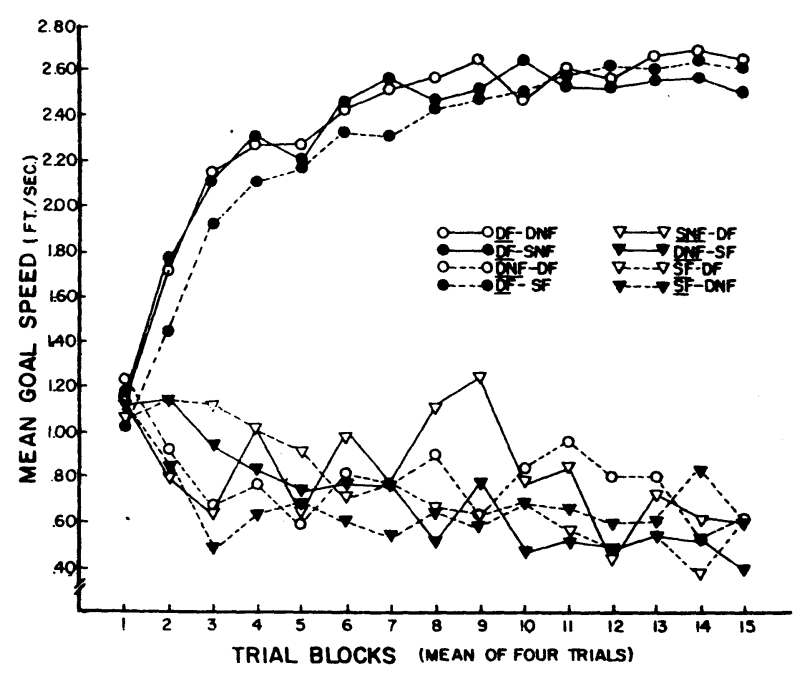

Figure 1. Mean goal speeds of the eight experimental groups during Phase I.

rapid extinction during Phase II is consistent with the expectation that rapid performance decrements should occur as $\mathrm{r}_{\mathrm{g}}$ - $\mathrm{sg}$ extinguishes as the result of omission of the UCS (food). Although subjects in Group DF-SF were presumably food satiated during Phase II, they continued to eat the food pellets in the goalbox, and the fact that their response speeds during Phase II were reliably faster than those of the satiated group from which food was removed again is consistent with the view that consummatory activity is the crucial underlying determinant of instrumental performance. Nevertheless, since the consummatory activity of Group DF-SF was gradually eliminated as a result of the satiation procedure, $r_{g}-s_{g}$ should have extinguished and there should have been concomitant decreases in $\mathrm{K}$ and in response speeds. The gradual decline in consummatory activity during Phase II was

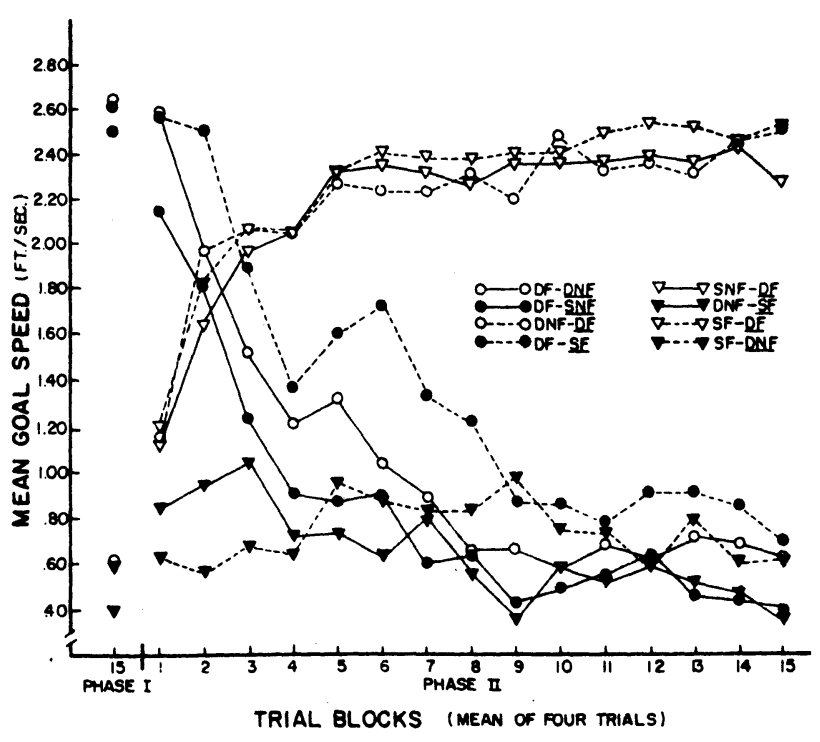

Figure 2. Mean goal speeds of the eight experimental groups during Phase II. 
in fact parallel by a decline in the speed with which subjects traversed the runway (Figure 2).

The effect of initial "satiated" or nonrewarded training upon Phase II performance. Inspection of Figure 2 indicates that there were no differences among the Phase II performance of the three groups which were shifted to the DF combination after having been trained under satiation and/or nonreinforcing combinations in Phase $I(F<1.0)$. Further, comparison of the performance of these three groups during Phase II with that of the three groups which were trained under the DF combination during Phase I (Figure 1) indicates a close resemblance between the two sets of performance curves in terms of rates of acquisition and asymptotic levels of responding. The training procedure for Group SF-DNF also provided a situation in which subjects might have "learned" during Phase I that food was available in the goalbox, even though they did not consume it, and might have demonstrated such "learning" during Phase II when they were deprived and the "reward" that had been in the goalbox was now relevant. This group, however, showed no substantial changes in responding from Phase I to Phase II (Figures 1 and 2). The performance of these four groups during Phase II therefore not only provides no evidence that subjects "learned" during Phase I that food was present in the goalbox, but they indicate that no benefit resulted from the nonreinforced training as well. These results thus again support the view that consummatory activity is the necessary event in instrumental reward conditioning and that a reward affects performance only when it is consumed.

Finally, subjects in Group DNF-SF apparently were food satiated during Phase II since eating appeared to have ceased prior to initiation of the daily trials and little or no eating appeared to occur in the carrying cages during the intertrial intervals. Yet, these subjects consumed approximately $25 \%$ of the pellets which were available in the goalbox throughout Phase II. Subjects in Groups SF-DF and SF-DNF, on the other hand, which received the same SF treatment during Phase $I$ and which had also been under a 10-day deprivation regimen prior to $P$ hase $I$ but which had received no prior training under deprivation, did not consume any of the available pellets during Phase I. A median test indicated that these groups differed reliably in terms of the proportion of the total available pellets consumed $\left(\chi^{2}=14.40, \mathrm{df}=2\right.$, $\mathrm{p}<.001)$. The consummatory activity which Group DNF-SF exhibited during Phase II might have been due to its initial experience in the runway while deprived, or since Group DNF-SF was somewhat older than Groups SF-DF and SF-DNF at the time of testing under satiation, the former group's consummatory activity during Phase II could have resulted from less complete food satiation for this group.

In any event, since subjects in Group DNF-SF did consume a portion of their available reward, $r_{g}-s_{g}$ should have become conditioned, and as a consequence $K$ and increases in response speeds should have developed. Inspection of Figure 2 indicates, however, that the performance of Group DNF-SF not only failed to improve during Phase II, but there was a tendency for response speeds to decline with trials. It should be noted further, however, that during Phase II subjects in this group slowly approached the goal dish to eat one or two of the available pellets and then turned away to engage in other nonconsummatory responses before their 20-sec goalbox confinement had elapsed. Black's (1969) "two-factor" analysis of instrumental conditioning predicts that even though $r_{g}-s_{g}$ may become conditioned and $\mathrm{K}$ may theoretically develop, the "apparent reinforcement value" of a consumed reward will be low if responses which are incompatible with the consummatory response also become conditioned to apparatus cues. Since in the present experiment, the consummatory response by subjects in Group DNF-SF was neither vigorous nor consistent, and thus the opportunity was provided for conditioning of such interfering responses, the present results seem consistent with Black's view.

\section{REFERENCES}

Black, R. W. On the combination of drive and incentive motivation. Psychological Review, 1965, 72, 310-316.

BLACK, R. W. Incentive motivation and the parameters of reward in instrumental conditioning. M. R. Jones (Ed.), Nebraska Symposium on motivation. Lincoln: University of Nebraska Press, 1969.

SEW ARD, J. P., \& Proctor, D. M. Performance as a function of drive, reward, and habit strength. American Journal of Psychology, 1960, 73, 448-453.

Seward, J. P., Shea, R. A., \& Davenport, R. H. Further evidence for the interaction of drive and reward. American Journal of Psychology, 1950, 73, 370-379.

Seward, J. P., Shea, R. A., \& Elkind, D. Evidence for the interaction of drive and reward. American Journal of Psychology, 1958, 71, 404-407.

SPENCE, K. W. Behavior theory and conditioning. New Haven: Yale University Press, 1956.

(Received for publication October 28, 1975.) 